\begin{tabular}{c} 
International Journal of Engineering \& Technology, 7 (3) (2018) 1696-1699 \\
International Journal of Engineering \& Technology \\
SPC \\
Website: $\begin{array}{c}\text { ww. sciencepubco.com/index.php/IJET } \\
\text { doi: } 10.14419 / \text { ijet.v7i3.15360 } \\
\text { Research paper }\end{array}$ \\
\hline
\end{tabular}

\title{
A novel approach for securing images using visual cryptographic scheme
}

\author{
Pushpa B. $\mathbf{R}^{1 *}$, Santhosh Kumar B. J ${ }^{2}$ \\ ${ }^{1,2}$ Department of Computer Science, Amrita School of Arts and Sciences, Mysuru Amrita Vishwa Vidyapeetham, India \\ *Corresponding author E-mail: preeths1@gmail.com
}

\begin{abstract}
Most applications require exchanging of information over insecure networks. The important issue concerned with respect to the images is to provide confidentiality and integrity. An efficient technique is proposed in order to provide protection to significant images through the digi-tal encryption scheme. Encryption before transmission is necessary for these images such that it makes sure that unauthorized person fails to access to any information. An efficient visual cryptographic approach is proposed for securing images by providing encryption during transferring an images or storing them. The technique proposed ensures that the images are inaccessible by unauthorized persons and also certifies confidentiality. In this proposed method to enhance security during data transmission across the network first the original image gets divided into multiple shares using logic operations and hence encryption technique is employed to these shares to maintain security. The binding of encryption and shares together called as encrypted shares are delivered and at the receiver side the reverse operation is carried out to retrieve back the original image. In the decryption process the decrypted share are layered to extract the actual image and if any shares are lost it is difficult to retrieve the information. The experimental result of the proposed method validates that the method will efficiently encrypt the image with a reduced amount of time without increasing the pixel size.
\end{abstract}

Keywords: Encryption; Decryption; Random Key Visual Cryptography; RSA; Images.

\section{Introduction}

Most of the organizations are preferring in exchanging digital information over internet that includes transferring of multimedia content such as files, video, audio and images etc .Hence it is significant to secure data over open and unsecured networks to ensure confidentiality of sensitive information. The proposed system can be applied for securing patient's medical information that is considered as sensitive and complex that needs the protection during the data storage, in the cloud and also during transmission across the hospitals. So cryptography techniques can use to provide protection of such data. In cryptography, encryption processes are applied to original data using a procedure to transform data to cipher text that is unreadable form. At the receiver end decryption process are carried out to get back the original data with the help of public or a private key.

There is a huge advancement in the field of Cryptography from the traditional approaches such as Caesar, Trifid and Vigenère ciphers to the current techniques that uses cipher and public key systems such as Diffie-Hellman etc. The cryptography procedure has been adapted to various kinds of digital file formats such as text, images etc. [1].One of the best-known techniques of visual cryptography has been credited to Moni Naor and Adi Shamir.

Visual Cryptography is a cryptographic technique used for encrypting the visual information like picture, text and other multimedia. The basic function of Visual Cryptography is that images are divided into several parts called shares. These shares are distributed among different participants across the media and it is not possible to acquire original information if any unauthorized person tries to access shares and to decrypt. During decryption these shares are stacked together to get the original image back.

\section{Literature survey}

Quist-AphetsiKester, MIEEE proposed a method for securing medical images. In this method first the input image was operated by a function to generate a secret key. The pixel (RGB) of the original image is shuffled based on the algorithm to which the secret key is applied to produce a cipher text. At the receiver end again a function is applied to decrypt the image. The proposed method was efficient in encryption and decryption process. It is observed that there is a slight pixel expansion in cipher image.

$R$. Norcen et al introduced a method to encrypt medical images. Here encryption is done in two levels based on AES algorithm. The first part encrypts a subset of bit planes of plain image data and the second it encrypts bit stream of the JPEG image. With this approach of selective bit plane encryption it is observed that up to $50 \%$ need to be encrypted whereas in the case of JPEG2000 bitstream encryption the protection of $20 \%$ data already provides a sustaining results.

Shankar K et al. here a new approach is proposed to provide great security for the images during transmission. In this approach the RGB value of the secret image is extracted. Each color component $\mathrm{R}, \mathrm{G}$ and $\mathrm{B}$ is represented in the form of matrix by applying some basic operation and a random key matrix is considered. Initially XOR operation of two matrices is carried out to create shares. Then AES algorithm and shares are binded together to get encrypted image and hence security is ensured and also reduces the fraudulent shares of the secret image.

Shital Bet al proposed visual cryptography to provide secured information sharing based on CMY colour based images that breaks down a secret image into several numbers of shares. The original 
secret images can be recovered by combining n-1 number of shares out of the $\mathrm{n}$ shares the original image can be retrieved. The proposed scheme ensures security as well. The method ensures confidentially that makes it impossible to grab the secret once the shares are generated and distributed across. This results in better performance as compared to RGB colour model.

\section{Limitation of the existing system}

It is been observed that the existing system proposed works efficiently in securing images using visual cryptographysuch that the shares are more secured and protected from the hacker who can alter to create fake shares but limitation found is that slight change in the ciphered image size and pixel values resulted in a change in the decryption result[1]. And also in the existing system the secrecy of the share is not maintained due to any other fake shares can easily insert or modified. The time required is more for encryption and decryption of shares. Hence our work is carried out in overcoming from these challenges.

\section{Proposed method}

The proposed scheme provides security of image by incorporating strong encryption and decryption technique. Hence, if someone tries to access any of the shares in unauthorized way it will be difficult to decrypt the information completely without the key and shares. This method imposes security and also the decrypted images are of the same size as original image.

The scheme is divided into three parts:

a) Generation of Shares

b) Encryption of original image

c) Decryption

The proposed method of visual cryptography is employed to send an original image from the sender to the receiver and achieving confidentiality and secrecy of the image. First the RGB color component is extracted from the original image. Each color R, G and $B$ are represented with values $(0-255)$ in the matrix form. Generate key matrix randomly (where $m=0$ to 255 ) based on the size of the RGB matrices. Then apply the XOR operation of Key matrix and the input image matrix. The resultant matrices are represented by $\mathrm{S} 1, \mathrm{~S} 2$ and $\mathrm{S} 3$ to generate the shares. Once all share generation process is done, RSA algorithm is implemented to encrypt the shares and to ensure security for the images and if any shares are missing then it is impossible to get back the original image. These encrypted shares are delivered to the receiving end.

During decryption process, the shares that are encapsulated are extracted by applying the RSA decryption process in order to get three shares and then the reverse operation of encryption is carried out .All these shares are stacked to get the actual image. The proposed method described above is represented in the figure 1.

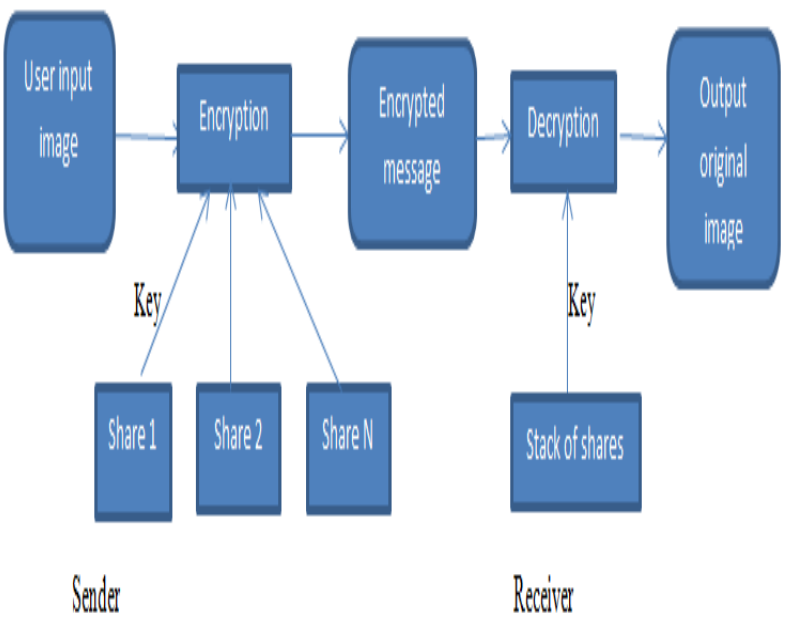

Fig. 1: Block Diagram of Visual Cryptography.

\subsection{Generation of shares}

Step 1: The R, G and B value is extracted from the original image that can be represented in a matrix form.

$\mathrm{B}=$

\begin{tabular}{llll}
\hline 113 & 109 & 105 & 102 \\
\hline 112 & 110 & 107 & 102 \\
111 & 112 & 106 & 105 \\
115 & 117 & 111 & 106 \\
135 & 136 & 135 & 114 \\
\hline
\end{tabular}

$\mathrm{G}=$

\begin{tabular}{llll}
\hline 105 & 101 & 99 & 96 \\
\hline 103 & 101 & 99 & 95 \\
103 & 102 & 97 & 97 \\
107 & 107 & 102 & 99 \\
129 & 130 & 128 & 108 \\
\hline
\end{tabular}

$\mathrm{R}=$

\begin{tabular}{llll}
\hline 64 & 63 & 58 & 56 \\
\hline 65 & 65 & 62 & 59 \\
63 & 69 & 63 & 62 \\
66 & 74 & 68 & 62 \\
84 & 89 & 88 & 72 \\
\hline
\end{tabular}

Step 2: Generate a random key matrix of the same size as the original image

$\mathrm{K}=$

\begin{tabular}{llll}
\hline 50 & 65 & 45 & 90 \\
\hline 40 & 45 & 82 & 88 \\
72 & 50 & 48 & 70 \\
92 & 60 & 95 & 86 \\
58 & 85 & 42 & 120 \\
\hline
\end{tabular}

Step 3: Applying the XOR operation on Key matrix and R,G and B matrix and store the result in $\mathrm{S} 1, \mathrm{~S} 2$ and $\mathrm{S} 3$.

$\mathrm{S} 1=$

\begin{tabular}{llll}
\hline 67 & 44 & 68 & 60 \\
\hline 88 & 67 & 57 & 62 \\
39 & 66 & 90 & 47 \\
47 & 73 & 48 & 60 \\
189 & 221 & 173 & 10 \\
\hline
\end{tabular}

\section{$\mathrm{S} 2=$}

\begin{tabular}{llll}
\hline 30 & 45 & 67 & 79 \\
\hline 80 & 45 & 50 & 23 \\
23 & 30 & 90 & 82 \\
58 & 70 & 50 & 12 \\
80 & 24 & 11 & 66 \\
\hline
\end{tabular}

$\mathrm{S} 3=$

\begin{tabular}{llll}
\hline 27 & 60 & 12 & 45 \\
\hline 70 & 54 & 34 & 20 \\
80 & 56 & 34 & 20 \\
53 & 45 & 60 & 70 \\
34 & 15 & 200 & 57 \\
\hline
\end{tabular}

Step 4: Shares that are generated in the previous step are subjected to encryption.

\subsection{Encryption of original image}

RSA algorithm, it is an RSA asymmetric cryptographic algorithm to encrypt and decrypt messages. It provides two different keys for 
cryptography. The keys are private and the public key. The public key is shared among everyone across the network, whereas the other key is maintained privately. The public key is used for encrypting the information and to decrypt the message the private key is mandatory.

The procedure for generating a public and private for encryption and decryption is illustrated below with example.

1) Initially the two discrete prime numbers $p$ and $q$ are chosen. The selected integers $\mathrm{p}$ and $\mathrm{q}$ should be chosen as random number with similar bit length.

2) Calculate $n=p * q$ where $n$ is used as the modulus for both the public and private keys that is expressed in bits.

3) An exponent e should be considered such that e must be an integer and not a factor of $n$. This creates public key.

4) To generate private key calculate $\emptyset(\mathrm{n})=(\mathrm{p}-1) *(\mathrm{q}-1)$

5) Compute $\mathrm{d}=(\mathrm{k} * \emptyset(\mathrm{n})+1) / \mathrm{e}$ such that $\mathrm{k}$ is an integer

6) Example

7) Let us assume $\mathrm{p}=7$ and $\mathrm{q}=11$ be the two prime numbers.

8) Computing theproduct of two primes $p * q=7 * 11=77$

9) Using Euler's totient function compute $\varphi(n)=(p-1) *(q-1)$ $=6 * 10=60$

10) Choose an integer e such that $1<\mathrm{e}<\varphi(\mathrm{n})$ where e and $\mathrm{n}$ are co-prime.

11) Considering $\mathrm{e}=7$ compute the value of dsuch that $(\mathrm{d} * \mathrm{e}) \% \varphi$ (n) $=1 . d=7-1 \bmod 60=43$

12) The resultant Public key $(e, n)=(7,77)$ and Private key is $(d, n)$ $=(43,77)$

13) To perform encryption, $\mathrm{m}=25$ is $\mathrm{c}=257 \bmod 77=53$

14) For decryption of $\mathrm{c}=53, \mathrm{~m}=5343 \bmod 77=25$

By applying above procedure the three shares S1, S2 and S3 gets encrypted and hence ensure more security and confidentially in an image. Even if the attacker hacks this shares it is impossible to retrieve the original image.

\subsection{Decryption}

First RSA decryption is applied, which exactly the reverse order of encryption. The resultant image is the image that was obtained during share generation. The three shares are operating in the following way to get the original image. First share S1 and the key matrix are XORed to obtain B component of the image and then share S2 and key matrix are combined to generate $\mathrm{G}$ component and $\mathrm{S} 3$ share is XORed with Key matrix to get $\mathrm{R}$ component then by combining al these three components $\mathrm{R}, \mathrm{G}$ and $\mathrm{B}$ values into single matrix to extract the original image.

\section{Experimental result}

The experiment is conducted for the proposed method using $\mathrm{c \#}$ language. The result obtained is presented in the figure 2 . To evaluate the performance of the proposed scheme number of experiments has been carried out with various image sizes types but every time secret color image is retrieved and the time taken to encrypt the shares is less than that of existing system.
(A)

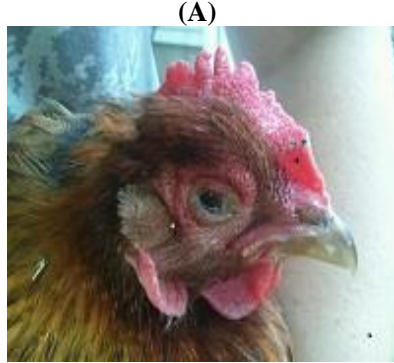

(C)

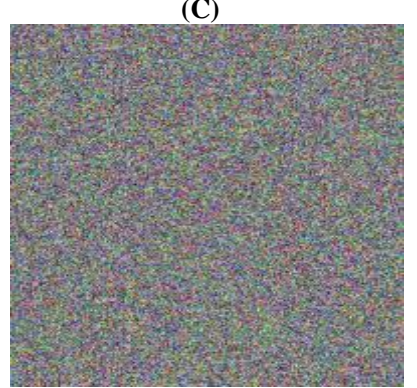

$(\mathbf{E})$

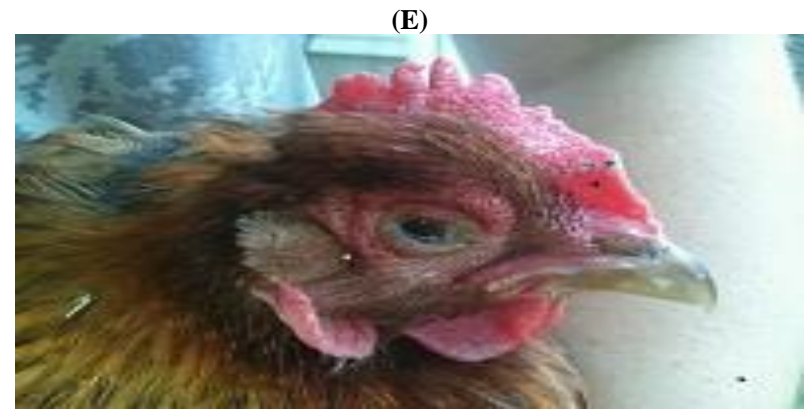

Fig. 2: A) Input Image. B) C) and D) Shares Encrypted Shares. E) Decrypted Original Image.

Comparison of time required for proposed method and the existing system

\begin{tabular}{lllll}
\hline Method & $\begin{array}{l}\text { Secu- } \\
\text { rity }\end{array}$ & Shares & $\begin{array}{l}\text { Encryp- } \\
\text { tion }\end{array}$ & $\begin{array}{l}\text { Decryp- } \\
\text { tion }\end{array}$ \\
\hline $\begin{array}{l}\text { Naor\& Shamir (Basic } \\
\begin{array}{l}\text { Ka) } \\
\text { Kaur \&KhemcHandani's }\end{array}\end{array}$ & $\begin{array}{l}\text { In- } \\
\text { crease }\end{array}$ & 2 & 0.82 & 0.85 \\
$\begin{array}{l}\text { In- } \\
\text { crease }\end{array}$ & 2 & 0.79 & 0.80 \\
Pcheme & $\begin{array}{l}\text { In- } \\
\text { crease }\end{array}$ & 3 & 0.86 & 0.84 \\
\hline
\end{tabular}

From the above table it is clear that the proposed work takes less time for encryption and decryption process.

The graph represents a slight pixel change in original image and the decrypted image.

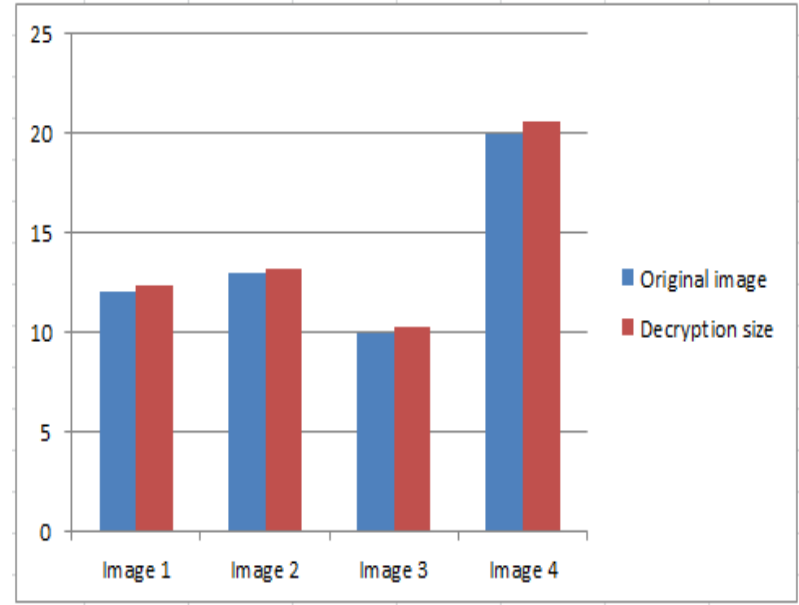




\section{Conclusion}

The proposed method coverts the original method to non-readable format. The encryption and decryption process was carried out effectively for the images A novel approach is implemented to generate a shares efficiently. It provides the combination of visual cryptography scheme to generate shares and encryption of shares is carried out to enhance security. There is no much pixel expansion in encrypted image. The shares are created by using random key and hence RSA encryption process is applied to increase the complexity. From the experimental analysis, it is observed that it occupies less memory utilization takes short span of time for computation.

\section{Acknowledgment}

The authors are grateful to Amrita Vishwa Vidyapeetham, Mysuru for the support given for doing the research. We are also thankful to our colleague for giving the valuable inputs in our work. We thank our family members and friends for their kind support.

\section{References}

[1] Quist-AphetsiKester, MIEEE “A Visual Cryptographic Encryption Technique for Securing Medical Images", in "International Journal of Emerging Technology and Advanced Engineering" Volume 3, Issue 6 , June 2013

[2] R. Norcen et al "Confidential storage and transmission of medical image data" inComputers in Biology and Medicine 33 (2003) 277 292. https://doi.org/10.1016/S0010-4825(02)00094-X.

[3] M. Sukumar Reddy et al "Visual Cryptography Scheme for Secre Image Retrieval “ in International Journal of Computer Science and Network Security, VOL.14 No.6, June 2014.

[4] Shankar K et al "Sharing a Secret Image with Encapsulated Shares inVisual Cryptography” inInternational Conference on Eco-friendly Computing and Communication Systems, (2015) 462 - 468.

[5] Shital B. Pawar, Prof.N.M.Shahane, "Visual Secret Sharing Using Cryptography" International Journal of Engineering Research (ISSN: 2319-6890) (online), 2347-5013(print) Volume No.3, Issue No.1, pp: 31-33.

[6] Ankush V et al. "Secret Sharing Based Visual Cryptography Scheme Using CMY Color Space" inInternational Conference on Information Security \& Privacy (ICISP2015), 11-12 December 2015,

[7] G. Elavarasi, Dr. M. Vanitha, "A Novel Method for Securing Medical Image Using Visual Secret Sharing Scheme”, International Journal of Engineering and Technology (IJET).

[8] A. Nandakumar, Harmya, P., Jagadeesh, N., and Anju, S. S., "A secure data hiding scheme based on combined steganography and visual cryptography methods", Communications in Computer and Information Science, vol. 191 CCIS, pp. 498-505, 2011

[9] A. Shamir, "How to share a secret", Commun. ACM, vol.22 (11) 1979, pp.612 - 613. https://doi.org/10.1145/359168.359176

[10] Mr.Reetesh.Rai "Secret Sharing based Visual Cryptography Scheme for color preservation using RGB Color Space" in International Journal of Computer Science and Information Technology \& Security (IJCSITS), ISSN: 2249-9555 Vol. 5, No5, and October 2015.

[11] C. Blundo, A. D. Santis, and M. Naor, "Visual cryptography for grey level images," Inf. Process. Lett, vol. 75, no. 6, pp. 255-259, 2000 https://doi.org/10.1016/S0020-0190(00)00108-3.

[12] SavitaPatil and JyotiRao,'Extended Visual Cryptography for Color Shares using Random Number Generator," International Journal of Advanced Research in Computer and Communication Engineering Vol. 1, pp 399-410 Issue 6, August 2012. 